\title{
A Remark on Helical Waveguides
}

\author{
Pavel Exner and Martin Fraas \\ Nuclear Physics Institute, Czech Academy of Sciences, 25068 Řez near Prague, \\ Doppler Institute, Czech Technical University, Břehová 7, 11519 Prague, Czechia \\ e-mail: exner@ujf.cas.cz,fraas@ujf.cas.cz
}

\begin{abstract}
Motivated by a proposal to create an optical helix-shaped waveguides for cold atoms and molecules, we discuss local perturbations which can create bound states in such a setting. This is known about a local slowdown of the twist; we show that a similar effect can result from a local tube protrusion or a change of the helix radius in correlation with its pitch angle.
\end{abstract}

\section{Introduction}

Geometrically induced bound states in tubular regions appear in various contexts - see, e.g., [DE95, CDFK05] and references therein. In particular, effects of waveguide twisting were discussed recently in [EKK05, EK05]. An independent strong motivation to look into such a problem came from a study [Bh07] which showed how helical waveguides for cold atoms and molecules can be created by means of two counter-propagating beams. Such waveguides do not have hard walls, of course, but it is known that spectrum-generating geometric mechanisms are robust - see, e.g., [EI01] - so it is useful to study such systems in a model which allows a mathematical treatment.

It was observed in [EK05] that slowing down the waveguide twist acts as an effective attractive potential. Since the mentioned optical waveguides allow for various modifications it is useful to look for other perturbations which could have a similar effect. We will discuss two of them. One is a local protrusion which is well known to create bound states in straight tubes [BGRS97], however, the proof needs to modified to fit to helical waveguides.

Another possible perturbation is a variation of the helix radius. Here it is more suitable to employ the formalism introduced originally in [DE95]. An exhaustive analysis would be complicated, however, and we restrict here to the situation of a thin waveguide when the cross section size is much smaller 
than the helix radius, paying attention to particular cases, those of a circular channel and of a flat "ribbon-shaped" one. In both cases we observe that a local reduction of the helix radius can induce a weakly bound state if the "pitch angle" is large enough, and in contrast, at small pitch angles localized states may appear for a local radius enlargement. At the same time, the crossover between the two regimes depends on the cross section.

\section{Helical channel Hamiltonians}

We will employ the notation of [EK05] which we extend to the case of varying cross-section and helix radius. Let $\omega$ be an open connected set in $\mathbb{R}^{2}$ we fix $t^{0}=\left(t_{2}^{0}, t_{3}^{0}\right) \in \omega$ and define

$$
\omega(\alpha)=\left\{l_{\alpha}(t):=\left(\alpha\left(t_{2}-t_{2}^{0}\right)+t_{2}^{0}, \alpha\left(t_{3}-t_{3}^{0}\right)+t_{3}^{0}\right): t \in \omega\right\} ;
$$

changing $\alpha$ means a radial scaling of the cross section $\omega$ w.r.t. the point $t^{0}$. Let further $\theta(s), \alpha(s)$ be differentiable functions from $\mathbb{R}$ to $\mathbb{R}$. We define the mapping $\mathcal{L}$ from $\mathbb{R} \times \omega$ to $\mathbb{R}^{3}$ by $\mathcal{L}:=\tilde{\mathcal{L}}(s, \tilde{t})$, where tilde ${ }^{1}$ marks the scaling (2.1) for $\alpha:=\alpha(s)$, i.e. $\tilde{t}=\left(\tilde{t}_{2}, \tilde{t}_{3}\right)=\left(\alpha(s)\left(t_{2}-t_{2}^{0}\right)+t_{2}^{0}, \alpha(s)\left(t_{3}-t_{3}^{0}\right)+t_{3}^{0}\right)$, and

$$
\tilde{\mathcal{L}}(s, t):=\left(s, t_{2} \cos \theta(s)+t_{3} \sin \theta(s), t_{3} \cos \theta(s)-t_{2} \sin \theta(s)\right) .
$$

We denote the $\mathcal{L}$-image of $\mathbb{R} \times \omega$ by $\Omega$. It is tube in $\mathbb{R}^{3}$ which is purely helical if the functions $\dot{\theta}(s), \alpha(s)$ are constant. We will be concerned with a quantum particle concerned within this tube assumimg that it has hard walls. Using appropriate units to get rid of unnecessary constants, we can then identify the particle Hamiltonian with the Dirichlet Laplacian $\mathrm{H}$ on $L^{2}(\Omega)$, i.e. the self-adjoint operator associated with the closed quadratic form

$$
\mathrm{Q}[\psi]:=\int_{\Omega}|\nabla \psi|^{2} \mathrm{~d} t \mathrm{~d} s, \quad \psi \in \mathcal{H}_{0}^{1}(\Omega) .
$$

The family of operators ${ }^{2}$ corresponding to given $\theta(s), \alpha(s)$ will be written as $\mathrm{H}(\alpha)$. In particular, $\mathrm{H}_{0}(\alpha)$ will denote the operator corresponding to $\theta(s)=$

\footnotetext{
${ }^{1}$ It would be appropriate, of course, to use $t$ indexed by the scaling parameter and to mark its dependence on $s$. Such a notation, however would be rather cumbersome and we believe that no confusion will arise. The tilde will be later used for functions on the scaled cross section $\omega(\alpha)$ as well.

${ }^{2}$ The function $\theta$ will be kept fixed so we do not write it explicitly in the operator symbol.
} 
$\beta_{0} s, \alpha(s)$, and $\mathrm{H}_{0}$ will refer to the purely helical tube, $\theta(s)=\beta_{0} s, \alpha(s)=$ $\alpha_{0}$. A simple substitution of variables shows that the quadratic form $Q_{0}(\alpha)$ associated with $\mathrm{H}_{0}(\alpha)$ is unitarily equivalent to

$$
\mathrm{Q}_{0}(\alpha)[\psi]=\int_{\mathbb{R}} \int_{\omega(\alpha(s))}\left(\left|\nabla_{t} \psi\right|^{2}+\left|\partial_{s} \psi+\beta_{0} \psi_{\tau}^{\prime}\right|^{2}\right) \mathrm{d} t \mathrm{~d} s,
$$

denoted for simplicity by the same symbol, where we have introduced the notation $f_{\tau}^{\prime}:=\left(t_{2} \partial_{t_{3}}-t_{2} \partial_{t_{2}}\right) f$ in accordance with [EK05].

Furthermore, the quadratic form (2.3) can transformed to other unitarily equivalent expressions supported by a straight tube (cylinder) is such a way that the geometric information is contained in the coefficients. Following [EKK05] we arrive at the quadratic form $\mathrm{q}^{\prime}$ [EKK05] acting on $L^{2}\left(\Omega_{0},|G|^{1 / 2}\right)$ as

$$
\mathbf{q}^{\prime}[\psi]:=\int_{\mathbb{R} \times \omega} \overline{\left(\partial_{i} \psi\right)} G^{i j}\left(\partial_{j} \psi\right)|G|^{1 / 2} \mathrm{~d} s \mathrm{~d} t,
$$

where we number the variables in such a way that $\left(\partial_{1}, \partial_{2}, \partial_{3}\right)=\left(\partial_{s}, \partial_{t_{2}}, \partial_{t_{3}}\right)$. By a straightforward computation we get for the metric tensor the expression

$$
G^{i j}=\left(\begin{array}{ccc}
1 & -\frac{h_{2}}{\alpha \alpha(s)} & -\frac{h_{3}}{\alpha(s)} \\
-\frac{h_{2}}{\alpha(s)} & \frac{1+h_{2}^{2}}{\alpha^{2}(s)} & \frac{h_{2} h_{3}}{\alpha^{2}(s)} \\
-\frac{h_{3}}{\alpha(s)} & \frac{h_{2} h_{3}}{\alpha^{2}(s)} & \frac{1+h_{3}^{2}}{\alpha^{2}(s)}
\end{array}\right),
$$

where

$$
\begin{aligned}
& h_{2}=\left(t_{2}-t_{2}^{0}\right) \dot{\alpha}(s)+\tilde{t}_{3} \dot{\theta}(s), \\
& h_{3}=\left(t_{3}-t_{3}^{0}\right) \dot{\alpha}(s)-\tilde{t}_{2} \dot{\theta}(s) .
\end{aligned}
$$

We can also pass to a quadratic form on the Hilbert space $L^{2}(\mathbb{R} \times \omega)$ without the additional weight $\left|G^{1 / 2}\right|$; this is achieved by putting [EKK05]

$$
\begin{aligned}
\mathbf{q}[\psi] & :=\mathbf{q}^{\prime}\left[|G|^{-1 / 4} \psi\right] \\
& =\left(\partial_{i} \psi, G^{i j} \partial_{j} \psi\right)+\left(\psi,\left(\partial_{i} F\right) G^{i j}\left(\partial_{j} F\right) \psi\right)-2 \operatorname{Re}\left(\partial_{i} \psi, G^{i j}\left(\partial_{j} F\right) \psi\right)
\end{aligned}
$$

where $F:=\log \left(|G|^{1 / 4}\right)$. If we finally plug in the metric tensor (2.6) we get

$$
\mathbf{q}[\psi]=\int \frac{1}{\alpha^{2}(s)}\left(\left|\alpha(s) \partial_{s} \psi-h_{2} \partial_{t_{2}} \psi-h_{3} \partial_{t_{3}} \psi-\dot{\alpha}(s) \psi\right|^{2}+\left|\nabla_{t} \psi\right|^{2}\right) \mathrm{d} s \mathrm{~d} t .
$$


After these preliminaries we shall formulate a theorem which will be proved in Section 4 below. We will consider helical tubes with a constant "pitch angle", $\theta(s)=\beta_{0} s$ for some $\beta_{0}>0$. Furthermore, we assume that outside a compact region $\Omega$ is purely helical; without loss of generality we may suppose that $\alpha(s)=1$ there so the unperturbed tube cross section is $\omega$.

Theorem 1 Suppose that $\alpha(s)-1$ is a nonzero continuous function, which is nonnegative and compactly supported. Let further $\omega, t^{0}$ be such that $\omega(\alpha) \subset$ $\omega\left(\alpha^{\prime}\right)$ holds for $\alpha \leq \alpha^{\prime}$, then the operator $\mathrm{H}_{0}(\alpha)$ has at least one eigenvalue below the threshold of the essential spectrum.

Remark We exclude here the case of a straight tube, $\beta_{0}=0$. Bound states induced by a local protrusion exists in such a situation also, however, one can use a more straightforward way to prove the claim - cf. [BGRS97].

\section{Spectrum of $\mathrm{H}_{0}$}

As in [EK05] our strategy is to regard $\mathrm{H}_{0}(\alpha)$ as a perturbation of $\mathrm{H}_{0}=$ $\mathrm{H}_{0}(1)$. The spectrum of the latter operator is purely absolutely continuous and covers the half-line $[E(1), \infty)$, where $E(\alpha)$ is the lowest eigenvalue of the operator

$$
\tilde{\mathrm{h}}(\alpha)=-\triangle_{D}^{\omega(\alpha)}-\beta_{0}^{2}\left(t_{2} \partial_{t_{3}}-t_{3} \partial_{t_{2}}\right)^{2}
$$

acting in $L^{2}(\omega(\alpha))$ (pay attention to the fact that this $\tilde{\mathrm{h}}(1)$ corresponds to $\mathrm{h}(0)$ of [EK05]). Moreover, the ground state is non-degenerate and the corresponding ground-state eigenfunction $\tilde{f}_{\alpha}(t)$ is strictly positive in $\omega(\alpha)$.

A simple substitution of variables given by $l_{\alpha}(2.1)$ allows us to pass to the unitarily equivalent operator $\mathrm{h}(\alpha)$ acting on $L^{2}(\omega)$, thus without a tilde. It acts as follows,

$$
\begin{aligned}
(\psi, \mathrm{h}(\alpha) \psi)= & \frac{1}{\alpha^{2}}(\psi, \mathrm{h}(1) \psi)-\frac{\beta_{0}^{2}}{\alpha^{2}} \int_{\omega}\left[\left|t_{2} \partial_{t_{3}} \psi-t_{3} \partial_{t_{2}} \psi\right|^{2}\right. \\
& \left.\quad-\left|\left(\alpha\left(t_{2}-t_{2}^{0}\right)+t_{2}^{0}\right) \partial_{t_{3}} \psi-\left(\alpha\left(t_{3}-t_{3}^{0}\right)+t_{3}^{0}\right) \partial_{t_{2}} \psi\right|^{2}\right] \mathrm{d} t .
\end{aligned}
$$

Next we ask about the dependence of $\mathrm{h}(\alpha)$ on $\alpha$. By $f_{\alpha}(t)$ we denote the

normalized ground-state eigenvector of $\mathrm{h}(\alpha)$, i.e. $\tilde{f}_{\alpha}\left(l_{\alpha}(t)\right)=f_{\alpha}(t)$. 
Lemma $2 f_{\alpha}(t), E(\alpha)$ are real-analytic functions of $\alpha$ in $(0, \infty)$. In particular, there are $E^{(1)}<0$ and $f^{(1)}(t)$ such that $E(\alpha)=E+(\alpha-1) E^{(1)}+o(\alpha-1)$ and $f_{\alpha}(t)=f(t)+(\alpha-1) f^{(1)}(t)+o(\alpha-1)$.

Proof: We rewrite and estimate the integral on the right-hand side of (3.2),

$$
\begin{aligned}
& \frac{\beta_{0}^{2}}{\alpha^{2}} \int_{\omega}\left[-\mid t_{2} \partial_{t_{3}} \psi\right.-\left.t_{3} \partial_{t_{2}} \psi\right|^{2} \\
&\left.+\left|\alpha\left(t_{2} \partial_{t_{3}} \psi-t_{3} \partial_{t_{2}} \psi\right)+(1-\alpha)\left(t_{2}^{0} \partial_{t_{3}} \psi-t_{3}^{0} \partial_{t_{2}}\right) \psi\right|^{2}\right] \mathrm{d} t \\
& \leq \frac{\beta_{0}^{2}}{\alpha^{2}}\left|1-2 \alpha^{2}\right| \int_{\omega}\left|t_{2} \partial_{t_{3}} \psi-t_{3} \partial_{t_{2}} \psi\right|^{2} \mathrm{~d} t \\
&+4 \frac{\beta_{0}^{2}}{\alpha^{2}}(1-\alpha)^{2} \int_{\omega}\left(\left|t_{2}^{0} \partial_{t_{3}} \psi\right|^{2}+\left|t_{3}^{0} \partial_{t_{2}} \psi\right|^{2}\right) \mathrm{d} t \\
& \leq C_{1}(\psi, \mathrm{h}(1) \psi)
\end{aligned}
$$

for some $C_{1}>0$, since the last integral can be estimated by (diam $\left.\omega\left\|\nabla_{t} \psi\right\|\right)^{2}$. It follows that the operators $\mathrm{h}(\alpha)$ form an analytic family type of type (B) - cf. [Ka, Sec. VII.4], note that it is a particular case of exercise 4.23. there. Hence the analyticity of $f_{\alpha}, E(\alpha)$ follows from finite multiplicity of the ground state; recall that in fact it is non-degenerate.

It remains to prove that $E^{(1)}<0$ which we will do using the minimax principle. Suppose that $\alpha>1$ and consider the test function obtained as a shifted ground state, $\tilde{\psi}(t):=f\left(t_{2}+(\alpha-1) a_{2}, t_{3}+(\alpha-1) a_{3}\right)$, where $f$ means here the lowest eigenfunction of $\mathrm{h}(1)$ extended to $\omega(\alpha)$ by zero. For small enough shifts $a_{2}, a_{3}$ we have $\left\{t_{2}+(\alpha-1) a_{2}, t_{3}+(\alpha-1) a_{3}\right\} \subset \omega(\alpha)$ for all $t=\left(t_{1}, t_{2}\right) \in \omega$, and consequently

$$
\begin{aligned}
(\tilde{\psi}, \tilde{\mathrm{h}}(\alpha) \tilde{\psi}) & =(f, \mathrm{~h}(1) f) \\
+ & 2(\alpha-1) \beta_{0}^{2} \int_{\omega}\left(t_{2} \partial_{t_{3}} f-t_{3} \partial_{t_{2}} f\right)\left(-a_{2} \partial_{t_{3}} f+a_{3} \partial_{t_{2}} f\right) \mathrm{d} t \\
& +(\alpha-1)^{2} \int_{\omega}\left|a_{2} \partial_{t_{3}} f-a_{3} \partial_{t_{2}} f\right|^{2} \mathrm{~d} t
\end{aligned}
$$

If the term linear in $(\alpha-1)$ does not vanish identically we are done. Suppose, on the contrary, that

$$
\int_{\omega}\left(t_{2} \partial_{t_{3}} f-t_{3} \partial_{t_{2}} f\right) \partial_{t_{3}} f \mathrm{~d} t=\int_{\omega}\left(t_{2} \partial_{t_{3}} f-t_{3} \partial_{t_{2}} f\right) \partial_{t_{2}} f \mathrm{~d} t=0
$$


holds, then we employ another test function, for instance one obtained by scaling, $\tilde{\psi}(t):=f\left(\frac{t_{2}-t_{2}^{0}}{\alpha}+t_{2}^{0}, \frac{t_{3}-t_{3}^{0}}{\alpha}+t_{3}^{0}\right)$. Using (3.2) we get

$$
\begin{aligned}
(\tilde{\psi}, \tilde{\mathrm{h}}(\alpha) \tilde{\psi})=E-\beta_{0}^{2} \int_{\omega}\left|t_{2} \partial_{t_{3}} f-t_{3} \partial_{t_{2}} f\right|^{2} \mathrm{~d} t-\left(1-\frac{1}{\alpha^{2}}\right) \int_{\omega}\left|\nabla_{t} f\right|^{2} \mathrm{~d} t \\
+\beta_{0}^{2} \int_{\omega}\left|t_{2} \partial_{t_{3}} f-t_{3} \partial_{t_{2}} f\right|^{2} \mathrm{~d} t+(\alpha-1)^{2} \frac{\beta_{0}^{2}}{\alpha^{2}} \int_{\omega}\left|t_{2}^{0} \partial_{t_{3}} f-t_{3}^{0} \partial_{t_{2}} f\right|^{2} \mathrm{~d} t \\
=E-2(\alpha-1) \int_{\omega}\left|\nabla_{t} f\right|^{2} \mathrm{~d} t+o(\alpha-1)
\end{aligned}
$$

and since $\int_{\omega}\left|\nabla_{t} f\right|^{2} \mathrm{~d} t>0$ we get the sought result.

\section{Helical channel with a protrusion}

Now we are in position to prove Theorem 1. By assumption the function $\alpha(s)-1$ is compactly supported, hence $\mathrm{H}_{0}(\alpha)-\mathrm{H}_{0}$ is relatively compact and $\sigma_{\text {ess }}\left(\mathrm{H}_{0}(\alpha)\right)=\sigma_{\text {ess }}\left(\mathrm{H}_{0}\right)$. Since we know the essential spectrum threshold, we can find eigenvalues below it using a variational estimate.

Since $\alpha(s)-1$ is supposed to be nonzero and non-negative, one can find an interval $\left(-s_{0}, s_{0}\right)$ within the support of this function on which the inequality $1+\varepsilon\left|s-s_{0}\right|<\alpha(s)$ holds for $\varepsilon$ small enough. It follows from the domain monotonicity of Dirichlet Laplacian that it is sufficient to establish existence of a bound state for $\alpha(s):=1+\varepsilon\left|s-s_{0}\right| \chi\left(-s_{0}, s_{0}\right)$. As usual in such cases we start constructing a trial function from then threshold-resonance of $\mathrm{H}_{0}$. Given $\delta>0, \varepsilon>0$ we put $\Psi_{\delta, \varepsilon}(s, t)=f_{\varepsilon}(t) \phi_{\delta}(s)$, where $f_{\varepsilon}$ is the groundstate eigenfunction of $\mathrm{h}\left(1+\varepsilon\left|s-s_{0}\right| \chi\left(-s_{0}, s_{0}\right)\right)$ and

$$
\phi_{\delta}(s)=\left\{\begin{array}{llr}
e^{\delta\left(s+s_{0}\right)} & \text { if } & s \leq-s_{0} \\
1 & \text { if } & -s_{0} \leq s \leq s_{0} \\
e^{-\delta\left(s-s_{0}\right)} & \text { if } & s>s_{0} .
\end{array}\right.
$$

We plug this expression into (2.10) and by a straightforward computation we 
get

$$
\begin{gathered}
\mathrm{q}\left[\Psi_{\delta, \varepsilon}\right]-E|| \Psi_{\delta, \varepsilon} \|^{2}=\delta+\int_{-s_{0}}^{s_{0}}\left(E\left(1+\varepsilon\left|s-s_{0}\right|\right)-E(1)\right) \mathrm{d} s \\
-2 \operatorname{Re} \int_{-s_{0}}^{s_{0}} \int_{\omega} \frac{\beta_{0}}{\alpha(s)}\left(\tilde{t}_{3} \partial_{t_{2}} f_{\varepsilon}-\tilde{t}_{2} \partial_{t_{3}} f_{\varepsilon}\right) \\
\times\left(\partial_{s} f_{\varepsilon}-\frac{\dot{\alpha}(s)}{\alpha(s)}\left(\left(t_{2}-t_{2}^{0}\right) \partial_{t_{2}} f_{\varepsilon}+\left(t_{3}-t_{3}^{0}\right) \partial_{t_{3}} f_{\varepsilon}-f_{\varepsilon}\right)\right) \mathrm{d} s \mathrm{~d} t \\
+\int_{-s_{0}}^{s_{0}} \int_{\omega}\left|\partial_{s} f_{\varepsilon}-\frac{\dot{\alpha}(s)}{\alpha(s)}\left(\left(t_{2}-t_{2}^{0}\right) \partial_{t_{2}} f_{\varepsilon}+\left(t_{3}-t_{3}^{0}\right) \partial_{t_{3}} f_{\varepsilon}-f_{\varepsilon}\right)\right|^{2} \mathrm{~d} s \mathrm{~d} t .
\end{gathered}
$$

Now we inspect the behavior of the expression for small $\varepsilon$ using Lemma 2 . Using $\dot{\alpha}(s) \sim \varepsilon \operatorname{sgn}(s)$ and $\partial_{s} f_{\varepsilon} \sim \varepsilon \operatorname{sgn}(s) f^{(1)}$ we obtain

$$
\begin{aligned}
& \mathrm{q}\left[\Psi_{\delta, \varepsilon}\right]-E\left\|\Psi_{\delta, \varepsilon}\right\|^{2}=\delta+\varepsilon \int_{-s_{0}}^{s_{0}} E^{(1)}\left|s-s_{0}\right| \mathrm{d} s \\
&-2 \varepsilon \beta_{0} \int_{-s_{0}}^{s_{0}} \int_{\omega}\left(t_{3}^{0} \partial_{t_{2}} f-t_{2}^{0} \partial_{t_{3}} f\right) \operatorname{sgn}(s) \\
& \times\left(f^{(1)}-\left(t_{2}-t_{2}^{0}\right) \partial_{t_{2}} f-\left(t_{3}-t_{3}^{0}\right) \partial_{t_{3}} f-f\right) \mathrm{d} t \mathrm{~d} s+o(\varepsilon) .
\end{aligned}
$$

The integral term vanishes due to the parity of the sign function; putting then $\delta=\varepsilon^{2}$ we arrive at

$$
\mathrm{q}\left[\Psi_{\delta, \varepsilon}\right]-E\left\|\Psi_{\delta, \varepsilon}\right\|^{2}=\varepsilon E^{(1)} s_{0}^{2}+o(\varepsilon) .
$$

Since $E^{(1)}$ is negative, it is sufficient to choose $\varepsilon$ small enough to conclude the proof.

\section{Thin helical tubes}

Next we look what happens if it is the radius rather than the cross section of the helical tube which is locally changed; for simplicity we restrict ourselves to small perturbations of a thin tube. To this aim it is more convenient to use the approach due to [GJ92] and [DE95] where the cross section is taken perpendicular to the tube axis. Let us stress that while helical tubes do not fall into the class of the asymptotically straight ones for which the existence of geometrically induced discrete spectrum was established in [DE95], the 
perturbation theory w.r.t. the tube radius developed there and in [CB96] remains nevertheless valid and we can use it here.

The generating curve of our thin channel will thus helix a varying radius,

$$
\tilde{\Gamma}(t)=(t, R(t) \cos \theta(t), R(t) \sin \theta(t)), \quad t \in \mathbb{R},
$$

whose image in $\mathbb{R}^{3}$ will be denoted $[\Gamma]$. With $\tilde{\Gamma}$ we conventionally associate its Frenet triad frame $(\mathbf{t}, \mathbf{n}, \mathbf{b})$ consisting of its tangent, normal, and binormal vectors. Furthermore, $\kappa, \tau$ will denote the curvature and torsion of $\tilde{\Gamma}$, respectively. We suppose that

$$
\theta(t)=\beta_{0} t
$$

and the radius is slightly changing according to

$$
R(t)=R_{0}+\varepsilon \delta(t),
$$

where $\delta(t)$ is a fixed (nonzero) $C^{2}$ smooth function of compact support and $\varepsilon$ is a small parameter by which we mean that $\varepsilon\|\delta\|_{\infty} \ll R_{0}$.

For further reference, let us first inspect the unperturbed helix, $\varepsilon=0$; the correspondent quantities will be indicated by the zero subscript. It is straightforward to check that for $\Gamma_{0}(t)=\left(t, R_{0} \cos \beta_{0} t, \sin \beta_{0} t\right)$ the Frenet triad is

$$
\begin{aligned}
& \mathbf{t}_{0}(t)=\left(\frac{1}{\sqrt{1+R_{0}^{2} \beta_{0}^{2}}},-\frac{R_{0} \beta_{0} \sin \beta_{0} t}{\sqrt{1+R_{0}^{2} \beta_{0}^{2}}}, \frac{R_{0} \beta_{0} \cos \beta_{0} t}{\sqrt{1+R_{0}^{2} \beta_{0}^{2}}}\right), \\
& \mathbf{n}_{0}(t)=\left(0,-\cos \beta_{0} t,-\sin \beta_{0} t\right), \\
& \mathbf{b}_{0}(t)=\left(\frac{R_{0} \beta_{0}}{\sqrt{1+R_{0}^{2} \beta_{0}^{2}}}, \frac{\sin \beta_{0} t}{\sqrt{1+R_{0}^{2} \beta_{0}^{2}}},-\frac{\cos \beta_{0} t}{\sqrt{1+R_{0}^{2} \beta_{0}^{2}}}\right) .
\end{aligned}
$$

The normal vector $\mathbf{n}_{0}(t)$ is perpendicular to the helix axis while the tangent $\mathbf{t}_{0}(t)$ and binormal $\mathbf{b}_{0}(t)$ contains with it nontrivial angles independent of $t$; the curvature and torsion are also constant and equal to

$$
\kappa_{0}=\frac{R_{0} \beta_{0}^{2}}{1+R_{0}^{2} \beta_{0}^{2}}, \quad \tau_{0}=\frac{\beta_{0}}{1+R_{0}^{2} \beta_{0}^{2}} .
$$

To use the above mentioned results [DE95] and [CB96] we have replace $t$ in the parametrization of $[\Gamma]$ by the arc length of the curve, $\Gamma(s):=\tilde{\Gamma}(t(s))$, where $t(s)$ is determined by the implicit equation

$$
s=\int_{0}^{t(s)}|\dot{\tilde{\Gamma}}(\tau)| \mathrm{d} \tau .
$$


The parametrization change makes, of course, little difference for the unperturbed helix where the two are mutually proportional, $t(s)=s\left(1+R_{0}^{2} \beta_{0}^{2}\right)^{-1 / 2}$.

We will consider two models of thin helix quantum waveguides corresponding to different cross-sections. The latter will a family $\omega(s)$ of bounded connected neighborhoods of zero, typically obtained by rotations of a fixed $\omega$ smooth w.r.t. $s$; the tube in question $\Omega \subset \mathbb{R}^{3}$ is then defined as the image of the map $s \mapsto \Gamma(s)+x_{2} \mathbf{n}+x_{3} \mathbf{b},\left(x_{2}, x_{3}\right) \in \omega(s)$, as $s$ runs through $\mathbb{R}$. The Hamiltonian is again the Dirichlet Laplacian on $\Omega$ denoted by $\mathrm{H}$ and the parametric description of $\Omega$ makes it possible to replace it by a unitary equivalent operator on the "straightened" waveguide. The two models we will be interested in are the following:

(i) The cross-section is two-dimensional, circular with $\Gamma$ at its centre, and perpendicular to the helix. In that case we can make $\omega$ fixed in the so-called Tang coordinate system which rotates around $\mathbf{t}$ w.r.t. the Frenet triad with the angular velocity $\tau$. In that case one achieves a full decoupling of the longitudinal and transverse coordinates in the "straightening" transformation, see [DE95] for details. The perturbation theory with respect to the circle radius developed there shows, in particular, that the bottom of the spectrum for a thin tube is determined - after subtracting the continuum threshold energy - by the one-dimensional Hamiltonian $T:=-\frac{\mathrm{d}^{2}}{\mathrm{~d}^{2} s}+V_{\text {eff }}^{\text {circ }}(s)$ with the effective potential

$$
V_{\mathrm{eff}}^{\mathrm{circ}}(s):=-\frac{1}{4} \kappa(s)^{2} \text {. }
$$

(ii) The optical waveguides which we use as a motivation [Bh07] are, however, far of a circular shape having a very elongated cross section the sizes of which in two principal directions may differ by as much as two orders of magnitude. In such a case it is appropriate to use an idealized description due to [CB96] in which the cross section is a onedimensional segment and $\Omega$ has thus form of a winding ribbon; in accordance with [Bh07] we suppose that the segment $\omega(s)$ is perpendicular to the helix axis. To achieve that, the function $\alpha$ describing the rotation of $\omega$ must be such that $(\mathbf{n} \cos \alpha-\mathbf{b} \sin \alpha)(s)$ is perpendicular the axis direction for any $s$, i.e. that the first component of this vector vanishes. By the analysis of [CB96] the weak-coupling problem is again described in the leading order by a one one-dimensional operator 
in which the effective potential (5.3) is replaced by

$$
V_{\mathrm{eff}}^{\mathrm{ribbon}}(s):=-\frac{1}{4} \kappa^{2}(s) \cos (\alpha(s))^{2}+\frac{1}{2}(\tau(s)-\dot{\alpha}(s))^{2} .
$$

Conditions for the existence of weakly bound states can be thus deduced from well-known properties of effective operator $-\frac{\mathrm{d}^{2}}{\mathrm{~d}^{2} s}+V_{\text {eff }}$ which has isolated eigenvalues if ${ }^{3}$ the potential is attractive in the mean, $\int_{\mathbb{R}}\left(V_{\text {eff }}(s)-E_{0}\right) \mathrm{d} s<0$, where $E_{0}:=\lim _{|s| \rightarrow \infty} V(s)$ ); in our case the limit obviously exists since the curvature and torsion are constant outside a compact set.

Let us look now what the above condition gives for the described geometries. Since the radius perturbation is weak by assumption, for the arc-length of perturbed helix we get from (5.2) the relations

$$
s=\sqrt{1+R_{0}^{2} \beta_{0}^{2}} t(s)+\mathcal{O}(\varepsilon), \quad t(s)=\frac{s}{\sqrt{1+R_{0}^{2} \beta_{0}^{2}}}+\mathcal{O}(\varepsilon)=: t_{0}(s)+\mathcal{O}(\varepsilon)
$$

since $\delta \in C^{2}$ by assumption, also the first two derivatives of $t(s)$ and $t_{0}(s)$ coincide up to $\mathcal{O}(\varepsilon)$. Then we can compute the geometric quantities which enter the above expressions for effective potentials; after a straightforward if tedious computation we get

$$
\begin{aligned}
& \kappa(s)=\kappa_{0}+\frac{\left(\beta_{0}^{2}-R_{0}^{2} \beta_{0}^{4}\right) \delta\left(t_{0}(s)\right)-\left(R_{0}^{2} \beta_{0}^{2}+1\right) \ddot{\delta}\left(t_{0}(s)\right)}{\left(1+R_{0}^{2} \beta_{0}^{2}\right)^{2}} \varepsilon+\mathcal{O}\left(\varepsilon^{2}\right), \\
& \tau(s)=\tau_{0}-2 \frac{R_{0}^{2} \beta_{0}^{4} \delta\left(t_{0}(s)\right)+\left(R_{0}^{2} \beta_{0}^{2}+1\right) \ddot{\delta}\left(t_{0}(s)\right)}{R_{0} \beta_{0}\left(1+R_{0}^{2} \beta_{0}^{2}\right)^{2}} \varepsilon+\mathcal{O}\left(\varepsilon^{2}\right), \\
& \tan \alpha(s)=-\frac{\dot{\delta}\left(t_{0}(s)\right)}{R_{0} \beta_{0} \sqrt{1+R_{0}^{2} \beta_{0}^{2}}} \varepsilon+\mathcal{O}\left(\varepsilon^{2}\right) .
\end{aligned}
$$

Comparing this results with the effective potential for the ribbon (5.4) we see that the terms linear in $\ddot{\delta}\left(t_{0}(s)\right)$ do not contribute to the integral in the condition $\int\left(V_{\text {eff }}(s)-E_{0}\right) \mathrm{d} s=0$ if $\delta$ is smooth as assumed, and thus the ribbon twisting described by the function $\alpha$ plays no role in the leading order. Computing the effective potentials explicitly with the help of the above formulae we arrive at the following conclusions:

\footnotetext{
${ }^{3}$ We leave out the critical case, $\int\left(V_{\text {eff }}(s)-E_{0}\right) \mathrm{d} s=0$, since using the effective operator we deal the leading order only.
} 
(i) for the circular tube we have

$$
\begin{aligned}
& V_{\text {eff }}^{\text {circ }}(s)=-\frac{R_{0}^{2} \beta_{0}^{4}}{4\left(1+R_{0}^{2} \beta_{0}^{2}\right)^{2}} \\
& +\frac{R_{0} \beta_{0}^{4}\left(R_{0}^{2} \beta_{0}^{2}-1\right) \delta(t(s))+R_{0} \beta_{0}^{2}\left(R_{0}^{2} \beta_{0}^{2}+1\right) \ddot{\delta}(t(s))}{2\left(1+R_{0}^{2} \beta_{0}^{2}\right)^{3}} \varepsilon+\mathcal{O}\left(\varepsilon^{2}\right) .
\end{aligned}
$$

Hence we can distinguish two cases: for a "steep" helix, $R_{0} \beta_{0}>1$, a weakly bound state occurs if $\int_{\mathbb{R}} \delta(t) \mathrm{d} t<0$, i.e. in the situation where the the helix radius is locally reduced. On the other hand, for a small pitch angle, $R_{0} \beta_{0}<1$ the bound state occurs if $\int_{\mathbb{R}} \delta(t) \mathrm{d} t>0$, i.e. if the radius is locally enhanced.

(ii) in the ribbon case we have

$$
\begin{aligned}
& V_{\mathrm{eff}}^{\mathrm{ribbon}}(s)=-\frac{R_{0}^{2} \beta_{0}^{4}-2 \beta_{0}^{2}}{4\left(1+R_{0}^{2} \beta_{0}^{2}\right)^{2}} \\
& \quad+\frac{R_{0}^{2} \beta_{0}^{4}\left(R_{0}^{2} \beta_{0}^{2}-5\right) \delta(t(s))+\left(1+R_{0}^{2} \beta_{0}^{2}\right)\left(R_{0}^{2} \beta_{0}^{2}-2\right) \ddot{\delta}(t(s))}{2 R_{0}\left(1+R_{0}^{2} \beta_{0}^{2}\right)^{3}} \varepsilon \\
& +\mathcal{O}\left(\varepsilon^{2}\right),
\end{aligned}
$$

and again we have two cases differing from the previous situation just by the critical value of the pitch angle. For $R_{0} \beta_{0}>\sqrt{5}$ the bound state occurs under the local "squeezing", while for $R_{0} \beta_{0}<\sqrt{5}$ we have to "inflate" the helix locally to achieve binding.

\section{Acknowledgments}

We are indebted to Dr. Bhattacharya for informing us about the results in [Bh07] prior to publication. The research was supported in part by the Czech Academy of Sciences and Ministry of Education, Youth and Sports within the projects A100480501 and LC06002.

\section{References}

[Bh07] M. Bhattacharya: Lattice with a twist: a helical waveguide for cold atoms and molecules, a talk and an article in preparation, http://meetings.aps.org/link/BAPS . 2007.MAR. P32.4 
[BGRS97] W. Bulla, F. Gesztesy, W. Renger, B. Simon: Weakly coupled bound states in quantum waveguides, Proc. Amer. Math. Soc. 125 (1997), 1487-1495.

[CDFK05] B. Chenaud, P. Duclos, P. Freitas: Geometrically induced discrete spectrum in curved tubes, Diff. Geom. Appl. 23 (2005), 95-105.

[CB96] I.J. Clark, A.J. Bracken: Effective potentials of quantum strip waveguide and their dependence upon torsion, J.Phys A: Math. Gen. 29 (1996), 339-348.

[DE95] P. Duclos, P. Exner: Curvature-induced bound states in quantum waveguides in two and three dimensions, Rev. Math. Phys. 7 (1995), $73-102$.

[EKK05] T. Ekholm, H. Kovařík, D. Krejčiřík: A Hardy inequality in twisted waveguides, Arch. Rat. Mech. Anal., to appear; math-ph/0512050.

[EI01] P. Exner, T. Ichinose: Geometrically induced spectrum in curved leaky wires, J. Phys. A: Math. Gen. 34 (2001), 1439-1450.

[GJ92] J. Goldstone, R.L. Jaffe: Bound states in twisting tubes, Phys. Rev. B45 (1992), 14100-14107.

[EK05] P. Exner, H. Kovařík: Spectrum of the Schroedinger operator in a perturbed periodically twisted tube, Lett. Math. Phys. 73 (2005), 183192.

[Ka] T. Kato: Perturbation Theory for Linear Operators, 3rd edition, Springer, Berlin 1984. 